\title{
Adenocarcinoma with Cartilaginous Metaplasia
}

National Cancer Institute

\section{Source}

National Cancer Institute. Adenocarcinoma with Cartilaginous Metaplasia. NCI

Thesaurus. Code C7685.

An adenocarcinoma characterized by the presence of cartilaginous metaplasia. 